\title{
FOREWORD
}

\section{Special Section on Advanced Transfer Technologies for the Next Generation Network}

The Next Generation Network (NGN) intends to integrate public phone service and data service networks seamlessly to create a multi-service network. To support new services and applications, the NGNs are expected to be a complex system interconnecting different technologies and various architectures across diverse software platforms. Therefore, there are numerous technical issues to be solved. Among these, one of the most important ones is a realization of high-speed transfer technologies with various functionalities for the NGNs; it also includes these issues of implementations of access, metro and core networks and QoS-enabled transport technologies. The Technical Committee on Communication Systems of the Communication Society in IEICE organized the special section to examine the current technical issues.

The Call for Papers attracted 43 submissions from academia and industry, and from China, Korea, Taiwan and U.S. as well as from Japan. The submission was evaluated with the help of the distinguished reviewers. The careful evaluation process has allowed us to accept only five high-quality papers. The acceptance rate for this special section was around $10 \%$.

The special section consists of one invited paper and four selected papers. The invited paper is an excellent introduction on the functional architecture of NGN, focusing on how the NGNs can be constructed and use various functionalities to provide the expected services.

Our sincere gratitude goes to all the authors who submitted their high-quality work to this special section. Finally, the Guest Editor-in-Chief would like to thank all reviewers and editorial committee members for investing their valuable time and providing rigorous assessment of the submitted papers.

Members of the Editorial Committee:

Guest Editors: Yoichi Maeda (NTT-AT), Hiromi Ueda (Tokyo University of Technology)

Guest Associate Editors: Satoru Aikawa (University of Hyogo), Tetsuro Fujii (NTT), Hiroyuki Fukuoka

(KDDI), Shin-ichiro Hayano (NEC), Tomohiro Ishihara (Fujitsu Labs), Hiroshi Ishii (NTT), Yoshihiro

Nakahira (OKI), Tomoaki Ohtsuki (Keio University), Naohiro Sakakida (Hitachi Communication Technology), Tetsuo Tsujioka (Osaka City University), Tetsuya Yokotani (Mitsubishi Electric Corp.)

Koichiro Wakasugi, Guest Editor-in-Chief

Koichiro Wakasugi(Member) received the B.E. degree in electrical engineering from Kyoto Institute of Technology in 1975, and the M.E. and Ph.D. degrees in electronic engineering from the Kyoto University in 1977 and 1981, respectively. Since 1980, he has been with Kyoto Institute of Technology. From 1985 to 1986, he was a Visiting Research Scientist with the National Institute of Standards and Technology, Boulder, CO. In 1987, he was a Visiting Professor at the Swiss Federal Institute of Technology, Zurich, Switzerland. He is currently Professor of Information Science and Director of the Center for Information Science, Kyoto Institute of Technology. His research interests are in the areas of information theory, communication theory and wireless networks. Prof. Wakasugi was General Secretary to the Board of Governors for the Society of Information Theory and its Applications from 1995 to 1996, and from 2006 to 2007. He has been serving as Chairman of the IEICE Technical Group on Communication Systems since 2006. 